\title{
Conserved quantities from pseudotensors and extremum theorems for angular momentum
}

\author{
Eduardo Nahmad-Achar $\dagger$ and Bernard F Schutz \\ $\dagger$ Institute of Astronomy, University of Cambridge, Cambridge CB3 OHA, UK \\ $\doteqdot$ Department of Applied Mathematics and Astronomy, University College, PO Box 78, \\ Cardiff CF1 1 XL, UK
}

Received 6 February 1986, in final form 14 November 1986

\begin{abstract}
A method for calculating pseudotensor-based conserved quantities for isolated systems in general relativity, independently of the asymptotic behaviour of the coordinate system used, is given. This allows the evaluation of concepts like energy, momentum and angular momentum in any coordinate system. The calculation is carried out for the Schutz-Sorkin gravitational Noether operator, a pseudotensorial vector operator which reduces to the familiar pseudotensors for particular choices of the vector fields; it is illustrated for the Kerr metric using várious fields and coordinates. We use this to prove a theorem of extremality of angular momentum for vacuum solutions of Einstein's equations, showing that any two of the following imply the third: (i) the metric is axisymmetric; (ii) Einstein's field equations are satisfied; (iii) the total angular momentum is an extremum against all perturbations satisfying a mild (and most reasonable) restriction. This theorem, valid for stationary and non-stationary metrics, is generalised to include matter fields, and, in particular, perfect fluids. A related theorem for extremising the angular momentum flux across a timelike hypersurface is also proved. This theorem provides an alternative way to solve the field equations for axisymmetric gravitational collapse.
\end{abstract}

\section{Introduction}

Variational principles for the structure of stationary but rotating stars in general relativity have been developed by Hartle and Sharp, Bardeen, and Schutz and Sorkin (see [1] and references therein). They are of the nature that a field is a solution of Einstein's equations if and only if it is an extremum of the total energy against a certain class of variations of the metric and fluid variables. Such principles can be used to construct stellar models, and they give us physical insight into such systems and their stability. However, energy is not the only conserved quantity one might have: there is also angular momentum. It is natural to ask whether variational principles also exist for angular momentum. Just as energy principles can be developed for stationary but non-axisymmetric systems [1], angular momentum principles may help one construct axisymmetric but non-stationary systems, such as collapsing stars. In this paper we develop such principles.

By definition, variational principles contemplate variations which are not necessarily solutions of Einstein's equations, and for which the asymptotic behaviour of the metric will therefore not necessarily contain information about the total energy or angular momentum of the configuration. For this reason, we base our variational principles 
on pseudotensorial measures of the conserved quantity. Although these are not coordinate independent [2], they are local, and they allow us to give the total energy or angular momentum of a configuration as an integral over it.

The problem with pseudotensors is that they must be evaluated in an asymptotically Lorentzian coordinate system (ALCS), which is inconvenient, i.e. one in which the metric takes the asymptotic form $\eta_{\mu \nu}+\mathrm{O}(1 / r)$ where here $\eta_{\mu \nu}$ is the Minkowski metric in rectangular coordinates. We want to free ourselves of this restriction by finding a method of evaluating pseudotensors that gives sensible results in any coordinate system.

Such a method has been given in detail in [3], together with some illustrations, and it will be briefly reviewed in $\$ 2$ below. In $\$ 3$ we will prove several extremum theorems for angular momentum, and comment on their physical interpretation. By comparing the results for energy and those for angular momentum, we will be able to prove a related theorem for the angular momentum flux across a timelike hypersurface $(\S 4)$.

\section{The quasi-covariant Noether operator for gravity}

In this section we will briefly review a way to compute conserved quantities in any coordinate system one chooses to work with, at infinity and locally (for full details see [3]). This is achieved by using a very simple trick, which consists essentially on working in special relativity.

Although this formalism applies to any pseudotensorial complex, we illustrate it here for the gravitational Noether operator $t^{\mu}{ }_{\nu}$ (introduced by Schutz and Sorkin in $[1]$ ), defined, for any vector field $\xi$, by

$$
8 \pi t_{N}^{\mu}{ }_{\nu} \cdot \xi^{\nu}:=-(-g)^{1 / 2} G^{\mu}{ }_{\nu} \xi^{\nu}+\frac{1}{2} \partial_{\alpha}\left(h^{\mu \alpha \nu \beta}{ }_{, \beta} \xi_{\nu}(-g)^{-1 / 2}\right)
$$

where

$$
h^{\mu \alpha \nu \beta}=(-g)\left(g^{\mu \nu} g^{\alpha \beta}-g^{\alpha \nu} g^{\mu \beta}\right)
$$

and $G^{\mu}{ }_{\nu}$ is the Einstein tensor. This pseudotensor has the advantages that it does not depend on second derivatives of the metric, it contains the Einstein [5] and the Landau-Lifshitz [6] pseudotensors as particular cases (for special choices of the vector field $\xi$ ), and when the field and matter equations are satisfied, the Schutz and Sorkin $\xi$ momentum, defined [1] by

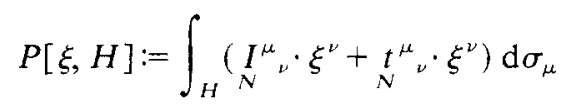

is conserved, and may be written as

$$
\begin{aligned}
P[\xi, H] & =\frac{1}{16 \pi} \int_{H} \partial_{\alpha}\left[h^{\mu \alpha \nu \beta \beta} \xi_{\nu}(-g)^{-1 / 2}\right] \mathrm{d} \sigma \\
& =\frac{1}{16 \pi} \oint_{\hat{\alpha} H} h_{, \beta}^{\mu \alpha \nu / \beta} \xi_{\nu}(-g)^{-1 / 2} \mathrm{~d} \Sigma_{\mu \alpha} .
\end{aligned}
$$

Here, $I_{N}^{\mu}{ }_{\nu}$ is the Noether operator for matter (a covariant generalisation of the so-called canonical stress-energy tensor), $H$ is a hypersurface with boundary $\partial H, \mathrm{~d} \sigma_{\mu}$ and $\mathrm{d} \Sigma_{\mu \alpha}$ are the coordinate volume and surface elements respectively in an ALCS. It is easy to 
show that, for the usual choices of vector fields, the quantity above gives the expected results at infinity for 4-momentum and angular momentum, in the geometries of Schwarzschild, Reissner-Nordström, Kerr-Newman, Vaidya, and many more.

The formalism for generalising (2.3) to give a quasi-covariant expression for conserved quantities (for isolated systems) is basically to change partial derivatives into flat-space covariant derivatives, as follows.

Let $\left(M, g_{\mu \nu}\right)$ be the spacetime manifold of an isolated gravitating system, together with an asymptotically Lorentzian coordinate system covering a neighbourhood of infinity. Associate to it the neighbourhood of infinity of the flat spacetime with Lorentz coordinates that correspond to the ALCS on $M$, and give it the metric of special relativity: $\left(M^{F}, \eta_{\mu \nu}\right)$. (Such a manifold $M^{F}$ always exists, given $M$.) Let $h$ be the one-to-one map between these neighbourhoods provided by the coordinates, and use it to bring the fields $g^{\mu \nu}$ and $\xi_{\nu}$ down to $M^{F}$. It is then easy to see that $h^{\mu \alpha \nu \beta}{ }_{\beta}(-g)^{-1 / 2} \xi_{\nu}$ is a tensor density of weight 1 in $M^{F}$, and that

$$
\begin{aligned}
P[\xi, H] & =\oint_{\partial H}(-\eta)^{-1 / 2} h^{\mu \alpha \nu \beta}{ }_{\mid \beta} \xi_{\nu}(-g)^{-1 / 2}(-\eta)^{1 / 2} \mathrm{~d} \Sigma_{\mu \alpha} \\
& =\oint_{\partial H} h^{\mu \alpha \nu \beta}{ }_{\mid \beta} \xi_{\nu}(-g)^{-1 / 2} \mathrm{~d} \Sigma_{\mu \alpha}
\end{aligned}
$$

(where a vertical bar denotes covariant differentiation WRT $\eta_{\mu \nu}$ and $\mathrm{d} \Sigma_{\mu \nu}$ is the coordinate surface element in $M^{F}$ ) gives the same value as (2.3) for the $\xi$ momentum, and is invariant under coordinate transformations in $M^{F}$. Since any transformation of coordinates in $M$ corresponds to one in $M^{F}$, the $\xi$ momentum (now defined as an integral on $M^{F}$ by (2.4)) is invariant under a transformation from an ALCS to any general curvilinear system in $M$ (cf [3] for details). We summarise this result in the following.

Proposition 2.1. Given any ALCS and a coordinate transformation from it to any curvilinear coordinate system, we can evaluate the $\xi$ momentum in this second system obtaining the same result as in the original ALCs.

Our procedure is still impractical because we must know the ALCS from which we start: arriving at a given curvilinear coordinate system from a second ALCS will give us for $P[\xi, H]$ its value in this second ALCs. (This is just the freedom we had at the beginning, consistent with the non-localisability of the $\xi$ momentum in general relativity.) To remove this ambiguity, we make use of the fact that $M$ is asymptotically flat in the sense that $g_{\mu \nu}=\eta_{\mu \nu}+\mathrm{O}\left(r^{-(1+\varepsilon) / 2}\right)$ asymptotically, for some $\varepsilon>0$, where $\eta_{\mu \nu}$ is the Minkowski metric and $r$ is a coordinate such that, to first order, surfaces of constant $r$ in every spacelike hypersurface have area $=4 \pi r^{2}$; and therefore its connection coefficients asymptotically approach those of the flat metric in some class of coordinate systems. For $M^{F}$ we choose that coordinate system, in this class, in which the connection coefficients exactly equal the leading order terms of those for $M$. This choice implicitly selects one particular ALCS, but we do not need to know how to reach it from our coordinates.

To illustrate the formalism, we can use it to evaluate the energy for Schwarzschild's and Kerr's geometries using different curvilinear coordinates and vector fields (a fuller account is given in [3]).

\subsection{Schwarzschild spacetime in Schwarzschild coordinates}

Use $\eta_{\mu \nu}$ in flat spherical polar coordinates, $\xi^{\mu}=(\partial / \partial t)_{\text {sch }}$, so that $\xi_{\nu}=(1-2 M / r) \mathrm{d} t$, $H=\{t=$ constant $\} \cap\left[r_{+}, R\right]$ where $r=r_{+}$is the horizon of the hole and $R$ is some 
radius greater than $r_{+}$. One then obtains, for the total energy (field plus hole) inside radius $R$,

$$
P[\xi, H]_{R} \equiv M
$$

independent of the radius. It is interesting to note that this agrees with the Penrose [4] mass for the Schwarzschild hole, as calculated by Tod [7], although this is defined in a completely different manner.

\subsection{Kerr spacetime in Boyer-Lindquist coordinates}

Again, we use $\eta_{\mu \nu}$ in flat spherical polar coordinates, $\xi^{\nu}=(\partial / \partial t)_{\mathrm{BL}}, H=\{t=$ constant $\} \cap$ $\left[r_{+}, R\right]$. The total energy inside a sphere of radius $R$ is then obtained as an exact expression in terms of hypergeometric functions [3]. At infinity one has, as expected, $P[\xi, H]=M$. Also, for $a=0: P[\xi, H] \equiv M$ in agreement with the previous result. For small values of $a$, one has

$$
P[\xi, H]_{R}=M-\frac{2 M a^{2}}{3 \Delta}+\frac{2 M^{2} a^{2}}{3 \Delta r}+\mathrm{O}\left(a^{4}\right) \quad \Delta=r^{2}-2 M r+a^{2} .
$$

This expression, as well as the full exact expression, is singular at the horizon, where $\Delta=0$. The problem arises from the combination of two facts: (i) the singularity of the coordinates themselves at the horizon; (ii) the fact that $\xi^{\mu}$ is spacelike at and near the horizon. That this is so may be seen in the following two examples.

\subsection{Kerr spacetime in Kerr-Schild coordinates}

These coordinates are obtained from the Boyer-Lindquist system via the transformation

$$
\mathrm{d} T=\mathrm{d} t-\frac{2 M r}{\Delta} \mathrm{d} r \quad \mathrm{~d} \Phi=\mathrm{d} \phi+\frac{2 M a r}{\Gamma \Delta} \mathrm{d} r
$$

with $\Gamma=r^{2}+a^{2}$. They reduce to the Eddington-Finkelstein coordinates for Schwarzschild spacetime.

Using $\eta_{\mu \nu}$ in spherical polar coordinates (note that this leads to a different ALCS than one would have got from Boyer-Lindquist coordinates), $\xi^{\mu}=(\partial / \partial T)$, and $H=$ $\{t=$ constant $\} \cap\left[r_{+}, r\right]$, one obtains an exact expression for $P[\xi, H]$ in terms of hypergeometric functions, which is well behaved everywhere and has the following limiting behaviour:

for $a=0: \quad P[\xi, H]=M+\left(2 M^{2} / r\right)$

far away from the horizon: $\quad P[\xi, H]=M+\left|\frac{2 M^{2}}{r}\right|-\left|\frac{2 M a^{2}}{3 r^{2}}\right|+\mathrm{O}\left(r^{-3}\right)$

on the horizon, to order $a^{2}$ :

$$
P[\xi, H]=M+\frac{2 M^{2} r_{+}^{3}}{\Gamma_{+}^{2}}-\frac{2 M a^{2} r_{+}^{2}}{3 \Gamma_{+}^{2}}+\frac{2 M^{2} a^{2} r_{+}}{3 \Gamma_{+}^{2}}+\mathrm{O}\left(a^{4}\right) .
$$

\subsection{Kerr spacetime in Boyer-Lindquist coordinates, second version}

This time consider $\eta_{\mu \nu}$ in spherical polar coordinates, $H=\{t=$ constant $\} \cap\left[r_{+}, r\right]$, and $\xi=e_{t}+\omega e_{\phi}$, where $\omega=-g_{r \phi} / g_{\phi \phi}$ is the angular velocity of a zero-angular-momentum 
observer ( $\mathrm{cf}$ [3] for motivation). Note that $\omega$ is not constant and so $\xi$ is not a Killing vector, but it is timelike outside the horizon and null on it.

Again, a well behaved exact expression is obtained for $P[\xi, H]$, with limiting behaviour:

far away: $\quad P[\xi, H]=M+\frac{2 M a^{2}}{3 r^{2}}+O\left(\frac{1}{r^{3}}\right)$

to order $a^{2}: \quad P[\xi, H]=\frac{M r^{4}}{\Gamma^{2}-\Delta a^{2}}\left[1-\frac{1}{3} \frac{\Delta a^{2}}{\left(\Gamma^{2}-\Delta a^{2}\right)}\right]$

$$
+\frac{1}{\Gamma^{2}-\Delta a^{2}}\left[\frac{2}{3} M a^{2} r^{2}-\frac{2}{3} M^{2} a^{2} r\right]+\mathrm{O}\left(a^{4}\right)
$$

Thus, sensible results can be obtained, even near black hole horizons, provided the coordinates and vector fields are chosen carefully. The flexibility that this gives us is exploited in the next section.

\section{Extremum theorems for angular momentum (at spatial infinity)}

The flexibility of evaluating conserved quantities in any coordinate system allows us to analyse properties that might help us in the study of isolated gravitating systems. We have in mind properties such as stability, or the very satisfaction of the field equations.

Extremality of conserved quantities has been proved by Schutz and Sorkin [1] in the following form, which applies to the simplest non-gravitational systems.

Theorem (Schutz and Sorkin). Given

$L$ : Lagrangian for an unconstrained variational principle,

$Q$ : set of field variables on which $L$ depends,

$\xi$ : vector field for which $\delta_{\xi} S=0$, where $S=\int L[Q] \mathrm{d}^{4} x$, then any two of the following imply the third:

(a) $Q$ is stationary WRT $\xi: £_{\xi} Q=0$

(b) $Q$ is a solution of the equations for the variational principle: $\delta L / \delta Q=0$.

(c) $P[\xi, H]$ is an extremum against all variations $\delta Q$ of compact support.

In the case of a gravitating system, any variation is likely to alter the asymptotic form of the metric as well, so $\delta g_{\mu \nu}$ would not have compact support and therefore the above theorem would not apply. Schutz and Sorkin were able to remove the constraint of compact support in the case of energy by assuming that, if the metric is written as $g_{\mu \nu}=\eta_{\mu \nu}+h_{\mu \nu}$ on the spacelike hypersurface $H$, then

$$
h_{\mu \nu}=\mathrm{O}\left(r^{-(1+\varepsilon) / 2}\right) \text { asymptotically, for some } \varepsilon>0 \text {. }
$$

We are here interested mainly in angular momentum, and we will assume that the metric has the following asymptotic behaviour in an AlCs near spatial infinity:

$g_{00}=1+\mathrm{O}(1 / r) \quad g_{i j}=-\delta_{i j}+\mathrm{O}(1 / r) \quad g_{0 k}=\mathrm{O}\left(1 / r^{1+\varepsilon}\right) \quad \varepsilon>0$.

For the sake of brevity, we will say that a metric is in class $A$ in a region $\Xi$ of spacetime if it satisfies (3.2) asymptotically in an ALCS on every spacelike hypersurface in that region. Note that class A is very general; only $g_{0 k}$ has been 'mildly' restricted, and it certainly allows for gravitational radiation to be present. We will also say that a spacelike hypersurface $H$ without boundary is asymptotically regular if it is asymptotically flat and $g_{\mu \nu}$ belongs to class A on $H$. 


\subsection{Stationary empty spacetimes}

Let us concentrate first on stationary empty spacetimes in class $\mathrm{A}$, and take $H$ to be an asymptotically regular spacelike hypersurface. Since we are interested in angular momentum, we take $\xi$ to be a vector field with the asymptotic behaviour

$$
\xi^{\mu}=x \delta_{2}^{\mu}-y \delta_{1}^{\mu} \text { (asymptotically on } H \text { ) }
$$

in an $\operatorname{ALCS}(T, x, y, z)$. We want to see how the $\xi$ momentum is altered when changing the metric asymptotically. To this end, consider another metric $\hat{g}_{\mu \nu}$ in class A and 'patch' it asymptotically to $g_{\mu \nu}$, i.e. consider the transformation

$$
g_{\mu \nu} \rightarrow t g_{\mu \nu}+(1-t) \hat{g}_{\mu \nu}
$$

with $0 \leqslant t \leqslant 1, t=1$ for $r<R, t=0$ for $r>2 R,\left|\partial_{k} t\right| \leqslant 2 / R$ and $R$ large. Then we have

$$
\begin{aligned}
& h_{\mu \nu} \rightarrow t h_{\mu \nu}+(1-t) \hat{h}_{\mu \nu} \\
& g_{, \alpha}^{\mu \nu} \rightarrow \operatorname{tg}_{, \alpha}^{\mu \nu}+(1-t) \hat{g}_{, \alpha}^{\mu \nu}+t_{, \alpha}\left(h^{\mu \nu}-\hat{h}^{\mu \nu}\right) \\
& \text { etc. }
\end{aligned}
$$

where $h_{\mu \nu}$ and $\hat{h}_{\mu \nu}$ are defined by

$$
\begin{aligned}
& g_{\mu \nu}=\eta_{\mu \nu}+h_{\mu \nu} \\
& \hat{g}_{\mu \nu}=\eta_{\mu \nu}+\hat{h}_{\mu \nu}
\end{aligned}
$$

(all the hatted quantities will refer to $\hat{g}_{\mu \nu}$ ).

Under the transformation (3.4), the change in the integrand of $(2.2)$ (here $I^{\mu}{ }_{\nu}=0$ since spacetime is empty, but $G^{\mu}{ }_{\nu}$ does not necessarily vanish since we are not assuming that Einstein's equations hold) takes a complicated form, but it may be shown that it can be reduced to

$$
P[\xi, H] \rightarrow P[\xi, H]_{r=R}+\frac{1}{16 \pi} \int_{r>R} h_{\beta}^{\mu \alpha \nu \beta} \frac{1}{\sqrt{-g}} \xi_{\nu, \alpha} \mathrm{d} \sigma_{\mu} .
$$

To show that the total $\xi$ momentum is unaltered under asymptotic changes in the metric, we need to show that the integral in (3.6) vanishes as $R \rightarrow \infty$. Noting that

$$
\begin{aligned}
& h_{, k}^{0 \alpha \nu k} \rightarrow t^{2} h_{, k}^{0 \alpha \nu k}+2 t t_{, k} h^{0 \alpha \nu \kappa}+(1-t)^{2} \hat{h}_{, k}^{0 \alpha \nu / \kappa}-2(1-t) t_{, k} \hat{h}^{0 \alpha \nu k}+t(1-t) \\
& \times\left[(-g)\left(g^{0 \nu} \hat{g}^{\alpha k}+g^{\alpha k} \hat{g}^{0 \nu}-g^{\alpha \nu} \hat{g}^{0 k}-g^{0 k} \hat{g}^{\alpha \nu}\right)\right]_{, k} \\
& +(1-2 t) t_{, k}\left[(-g)\left(g^{0 \nu} \hat{g}^{\alpha k}+g^{\alpha k} \hat{g}^{0 \nu}-g^{\alpha \nu} \hat{g}^{0 k}-g^{0 k} \hat{g}^{\alpha \nu}\right]\right.
\end{aligned}
$$

and

$$
\begin{aligned}
\xi_{\nu, \alpha} \rightarrow t_{, \alpha}\left[x \left(g_{2 \nu}\right.\right. & \left.\left.-\hat{g}_{2 \nu}\right)-y\left(g_{1 \nu}-\hat{g}_{1 \nu}\right)\right]+t\left[\delta_{1 \alpha}\left(g_{2 \nu}-\hat{g}_{2 \nu}\right)\right. \\
& \left.-\delta_{2 \alpha}\left(g_{1 \nu}-\hat{g}_{1 \nu}\right)\right]+\left[\delta_{1 \alpha} \hat{g}_{2 \nu}-\delta_{2 \alpha} \hat{g}_{1 \nu}+t\left[x\left(g_{2 \nu}-\hat{g}_{2 \nu}\right)_{, \alpha}-y\left(g_{1 \nu}-\hat{g}_{1 \nu}\right)_{, \alpha}\right]\right] \\
& +\left[x \hat{g}_{2 \nu, \alpha}-y \hat{g}_{1 \nu, \alpha}\right]
\end{aligned}
$$

we see that the integral in (3.6) vanishes as $R \rightarrow \infty$ iff

$$
\left\langle\varepsilon^{3 i k} n_{i} \hat{g}_{O k}\right\rangle=\left\langle\varepsilon^{3 i k} n_{i} g_{O h}\right\rangle+\mathrm{O}\left(\frac{1}{r^{2+k}}\right) \text { (asymptotically) }
$$


for some $\varepsilon>0$, where $\langle\ldots\rangle$ denotes integration over the unit sphere, i.e. $\langle f\rangle=\oint f \mathrm{~d} \Omega=$ $\oint f \sin \theta \mathrm{d} \theta \mathrm{d} \phi$, and $n$ is the unit radial vector: $n_{i}=\partial r / \partial x^{i}$. In other words, the integral in (3.6) will vanish as $R \rightarrow \infty$, i.e. the $\xi$ momentum will be unaltered under an asymptotic metric change, if the above average is equal asymptotically for both metrics up to second order in $r^{-1}$. (The index ' 3 ' arises explicitly in (3.8) because of the form we have chosen for the vector field $\xi$, equation (3.3).)

We then have the following extension of the Schutz and Sorkin theorem.

Theorem 3.1. Any axisymmetric stationary empty spacetime is a solution to Einstein's equations in some region iff it is an extremum of the angular momentum against all variations satisfying (3.8) on each spacelike hypersurface in that region. When the region extends out to infinity, the metric is assumed to have the asymptotic fall-off given by (3.2).

By analogy with [1], the stronger version in the form "any two of the three statements imply the third' is also obtained. The only proof that has been strengthened is $(a)+(b) \rightarrow(c)$, by showing that any variation of non-compact support which satisfies (3.8) affects the angular momentum in just the same way as one of compact support.

Actually, our result so far is stronger than stated, for stationarity was only used asymptotically. Thus, provided that $P[\xi, H]$ is well defined for a certain non-stationary spacetime, we have shown that it is unchanged under the transformation (3.4) if this spacetime is asymptotically stationary. Theorem 3.1 is also valid for this more general case.

What is the meaning of the restriction (3.8) on the variations? One can calculate this quantity for a few known simple spacetimes and see that it is essentially the angular momentum of the spacetime. In fact, that it is essentially the angular momentum for metrics that solve the field equations can be seen in linearised theory, where one has

$$
\begin{aligned}
g_{0 j}(r, t)=h_{0 j}(r, t)=-4 \int \frac{T_{0 j}(\bar{r}, t-|r-\bar{r}|)}{|r-\bar{r}|} \mathrm{d}^{3} \bar{x} \\
=-\frac{4}{r} \int T_{0 j} \mathrm{~d}^{3} \bar{x}-\frac{4}{r^{3}} \int T_{0 j} x^{i} \bar{x}_{i} \mathrm{~d}^{3} \bar{x} \\
+ \text { mass quadrupole terms and their derivatives, which we neglect }
\end{aligned}
$$

where the integrals are taken over the sources. Now the first of these integrals does not contribute to the average in (3.8), and the second gives

$$
\begin{aligned}
\left\langle\varepsilon^{3 k j} n_{k} g_{0 j}\right\rangle & \approx-\frac{4}{r^{3}}\left(\int T_{0 j} \bar{x}_{i} \mathrm{~d}^{3} \bar{x}\right) \int n_{k} x^{i} \mathrm{~d} \Omega \varepsilon^{3 k j} \\
& =-\frac{4}{r^{4}}\left(\int T_{0 j} \bar{x}_{i} \mathrm{~d}^{3} \bar{x}\right) \varepsilon^{3 k j} 4 \pi r^{2} \delta_{k}^{i} \\
& =-\frac{16 \pi}{r^{2}}\left(\int T_{0 j} \bar{x}_{i} \mathrm{~d}^{3} \bar{x}\right) \varepsilon^{3 i j} \\
& =\frac{16 \pi}{r^{2}} \text { [angular momentum]. }
\end{aligned}
$$

However, for spacetimes which do not solve Einstein's equations, the integral over the 3 -surface $H$ does not convert to an integral over $\partial H$ and so $\left\langle\varepsilon^{3 k j} \eta_{k} g_{0 j}\right\rangle$ is not the 
angular momentum of the metric as we have defined it. Thus, when comparing a solution to the field equations with neighbouring non-solutions, the variations are allowed to range over spacetimes with different angular momentum, but if other solutions are covered by the variation, then the theorem applies only if these other solutions have the same value of the angular momentum (at spatial infinity). In this sense general variations are allowed, for the 'restriction' is the obvious one: the theorem is singling out that metric (out of a whole set of neighbouring metrics) which satisfies Einstein's equations, and it is implying that this metric is an extremum of the angular momentum; if two (or more) metrics in the set solve Einstein's equations, they cannot but have the same angular momentum.

\subsection{Non-stationary electrovac spacetimes}

The results above may be generalised to non-stationary metrics in class $\mathrm{A}$. We assume here that matter fields are not present and only allow gravitational and electromagnetic radiation in the spacetime. (Matter fields will be considered in the next section.) In this case, however, one must first impose conditions on the metric coefficients which guarantee that the angular momentum of the spacetime is well defined at spacelike infinity (i.e. that relevant integrals do indeed converge).

A careful analysis of orders of magnitude reveals that these conditions are

$$
\begin{aligned}
& \left\langle\varepsilon^{3 i k} n_{i} g_{k j}\right\rangle=\mathrm{O}\left(\frac{1}{r^{1+\varepsilon}}\right) \\
& \left\langle\varepsilon^{3 i k} n_{i} g_{k j} n_{l} g^{l j}, 0\right\rangle=\mathrm{O}\left(\frac{1}{r^{3+\varepsilon}}\right)
\end{aligned}
$$

asymptotically in an ALCS, for some $\varepsilon>0$, and where the index ' 0 ' refers to time.

When we compute the variation of the angular momentum under an asymptotic patching of the form (3.4), in the same manner as for the stationary case but now allowing for time derivatives of all the quantities involved, we see that the conditions for this variation to be zero are precisely that the equations (3.8) and (3.11) be satisfied, i.e. we have the following.

Theorem 3.2. Any axisymmetric vacuum solution to Einstein's equations is an extremum of the angular momentum against all variations of the metric satisfying (asymptotically)

$$
\left\langle\varepsilon^{3 i k} n_{i} \hat{g}_{0 k}\right\rangle=\left\langle\varepsilon^{3 i k} n_{i} g_{0 k}\right\rangle+\mathrm{O}\left(\frac{1}{r^{2+\varepsilon}}\right)
$$

and

$$
\begin{aligned}
& \left\langle\varepsilon^{3 i k} n_{i} g_{k j}\right\rangle \sim\left\langle\varepsilon^{3 i k} n_{i} \hat{g}_{k j}\right\rangle=\mathrm{O}\left(\frac{1}{r^{1+\varepsilon}}\right) \\
& \left\langle\varepsilon^{3 i k} n_{i} g_{k j} n_{l} g_{, 0}^{l j}\right\rangle \sim\left\langle\varepsilon^{3 i k} n_{i} \hat{g}_{k j} n_{i} \hat{g}^{l j}{ }_{, 0}\right\rangle=\mathrm{O}\left(\frac{1}{r^{3+\varepsilon}}\right) .
\end{aligned}
$$

(When the region under consideration extends out to infinity, the metric is assumed to have the asymptotic fall-off given by (3.2).)

As before, the other two implications are also obtained. Equation (3.8) was expected from the stationary case, and we have already seen its meaning above. Equations 
(3.12) guarantee that the angular momentum is well defined for both $g_{\mu \nu}$ and $\hat{g}_{\mu \nu}$, and for its mixture $t g_{\mu \nu}+(1-t) \hat{g}_{\mu \nu}$. One may ask how strong these conditions are, i.e. how much should one restrict the metric in order to be able to talk about angular momentum and work with it?

Equation (3.11a) is actually a mild restriction on the metric coefficients, and it is always satisfied in linearised theory. To see how strong (3.11b) is, let us look at the Cauchy problem for asymptotically flat spacetimes in general relativity.

In order to construct gravitational initial data $(\Sigma, \gamma, K)$ (where $\Sigma$ is an initial spacelike hypersurface, and $\gamma$ and $K$ are two 2 -index tensor fields) that satisfy the momentum constraints or initial-value equations (which are just the Gauss-Codazzi equations expressing the necessary and sufficient conditions that $\gamma$ and $K$ are indeed the metric and extrinsic curvature of the submanifold $\Sigma$ of a spacetime that satisfies Einstein's equations, plus the constraints for the electromagnetic field which are of the form (in Gaussian units): $D_{i} E^{i}=4 \pi q, D_{i} B^{i}=0$ ) one assumes [8] that

$$
\begin{aligned}
& K_{j}^{i}=\mathrm{O}\left(1 / r^{2}\right) \\
& h_{j}^{i}=\mathrm{O}(1 / r)
\end{aligned}
$$

where $\gamma_{i j}=\eta_{i j}+h_{i j}$. (When electromagnetic fields are present, one must ask for the energy and momentum densities $\left(\rho, j_{i}\right)$ of the field to fall off fast enough to render the total energy and momentum finite.) These conditions ensure that at least the energy and the linear momentum associated to the initial data set (which may contain matter fields and currents) are well defined. To guarantee that these restrictions are preserved in the evolution of the initial data, one asks for the (cf [8])

$$
\begin{aligned}
& \text { lapse function } \equiv \alpha \equiv\left(g^{00}\right)^{-1 / 2}=1+\mathrm{O}\left(r^{-1}\right) \\
& \text { shift function } \equiv \beta^{a} \equiv g^{0 a}=\mathrm{O}\left(r^{-1}\right) .
\end{aligned}
$$

In our case, we have $h^{k}{ }_{j}=\mathrm{O}\left(r^{-1}\right)$ in agreement with $(3.14), \alpha=1+\mathrm{O}\left(r^{-1}\right)$ in agreement with (3.15), and $\beta^{a}=\mathrm{O}\left(1 / r^{1+\varepsilon}\right)$ which is slightly stronger than (3.16). In other words, our requirements on $g_{\mu \nu}$ agree with (3.14) and guarantee that (3.13) and (3.14) are preserved under the evolution of the data. What is (3.13)?

If $\hat{n}$ is the unit normal vector field to $\Sigma$, we have

$$
K_{\alpha \beta}=\frac{1}{2} \gamma_{\alpha}^{\lambda} \gamma_{\beta}^{\mu}\left(\nabla_{\lambda} \hat{n}_{\mu}+\nabla_{\mu} \hat{n}_{\lambda}\right) \text {. }
$$

When $\Sigma$ is given by $\Sigma=\left\{x^{0}=0\right\}$ this reduces to

$$
K_{i j}=-\frac{1}{2}\left(\nabla_{j} \hat{n}_{i}+\nabla_{i} \hat{n}_{j}\right)=-\alpha \Gamma_{i j}^{0}
$$

Now,

$$
K_{j}^{i}=g^{i \alpha} K_{\alpha j}=\left(g^{00}\right)^{-1 / 2} \Gamma^{0}{ }_{k j} \delta^{k i}+\mathrm{O}(1 / r)
$$

and therefore (3.13) implies

$$
g_{i j, 0}=\mathrm{O}\left(1 / r^{2}\right)
$$

which is a stronger condition than the one we have used $\left(g_{i, 0}=O\left(r^{-1}\right)\right)$ for the proof of the theorems above. In fact, the restriction $(3.11 b)$ arises from the condition of convergence of the integral

$$
\int_{H}(-g) g^{00} g^{k l}{ }_{, 0} \xi_{l, k}(-g)^{-1 / 2} \mathrm{~d}^{3} x
$$

but when (3.20) is satisfied, restriction $(3.11 b)$ is no longer needed! In other words, the conditions used for the initial-value problem to be well defined are consistent with 
and imply our restriction $(3.11 b)$ on the metric coefficients for the angular momentum to be well defined, so that only $(3.11 a)$ is needed. In this sense, our restrictions do not limit ourselves significantly in the choice of metrics with which we are able to work.

\subsection{Extension to matter fields}

We will now generalise the above results to include any matter field of compact support in the spacetime, and, in particular, perfect fluids.

Writing the action as $S=S_{G}+S_{F}$, where $S_{G}$ is the action for the gravitational field and $S_{F}$ is that for the matter field, the vanishing of its variation in any region $\Xi$ of spacetime takes the form [1]

$$
\begin{gathered}
-\frac{1}{16 \pi} \int_{\equiv}\left(G^{\alpha \beta}-8 \pi T^{\alpha \beta}\right)(-g)^{1 / 2} £_{\xi} g_{\alpha \beta} \mathrm{d}^{4} x+\int_{\Xi} I_{\beta ; \alpha}^{\alpha} \xi^{\beta} \mathrm{d}^{4} x \\
=\oint_{\partial \Xi}\left(t^{\alpha}{ }_{\beta} \xi^{\beta}+I^{\alpha}{ }_{\beta} \xi^{\beta}\right) \mathrm{d} \sigma_{\alpha x}
\end{gathered}
$$

where $T^{\alpha \beta}$ is the energy-momentum tensor of the matter, and $I_{\beta}^{\alpha}$ its energy-momentum tensor density (which is a Noether operator for the matter field).

The total $\xi$ momentum $P[\xi, H]=\int_{H}\left(t_{N}^{\mu}{ }_{\nu} \cdot \xi^{\nu}+I_{\nu}^{\mu} \cdot \xi^{\nu}\right) \mathrm{d} \sigma_{\mu}$ will be conserved if either $(a)$ Einstein's equations are satisfied; or $(b) \xi$ is a Killing vector of $g_{\alpha \beta}$ and the matter's $\xi$ momentum is locally conserved: $\partial_{\beta}\left(\xi^{\alpha} I_{c z}^{\beta}\right)=0$. The first variation of $(3.22)$ is

$$
\begin{gathered}
\int_{\Xi}\left(-\frac{1}{16 \pi}\left(G^{\alpha \beta}-8 \pi T^{\alpha \beta}\right)(-g)^{1 / 2} £_{\xi} \delta g_{\alpha \beta}-\frac{1}{16 \pi} \delta\left[\left(G^{\alpha \beta}-8 \pi T^{\alpha \beta}\right)\right.\right. \\
\left.\left.\times(-g)^{1 / 2}\right] \mathfrak{E}_{\xi} g_{\alpha \beta}+\delta\left(I_{\alpha ; \beta}^{\beta}\right) \xi^{\alpha}\right) \mathrm{d}^{4} x \\
=\delta \int_{\partial \equiv{ }_{N}\left[t^{\mu} \xi^{\nu}+I_{\nu}^{\mu} \xi^{\nu}\right] \mathrm{d} \sigma_{\mu} .}
\end{gathered}
$$

We then have the following.

Theorem 3.3. Let $H$ be any asymptotically regular, spacelike hypersurface in a region $\Xi$ of spacetime, and let $\xi^{\mu}=x \delta_{2}^{\mu}-y \delta_{1}^{\mu}$ asymptotically in an ALCS. Then any two of the following imply the third.

(a) Axisymmetry: $\mathfrak{E}_{\xi g_{\alpha \beta}}=0$.

(b) Einstein's equations are satisfied in $\Xi: G^{\alpha \beta}-8 \pi T^{\alpha \beta}=0$.

(c) The angular momentum $P[\xi, H]$ of every such $H$ is an extremum against all variations of the metric and matter fields that satisfy

$$
\begin{aligned}
& \delta\left(I_{\alpha ; \beta}^{\beta}\right) \xi^{\alpha}=0 \\
& \left\langle\varepsilon^{3 i k} n_{i} \delta g_{0 k}\right\rangle=\mathrm{O}\left(\frac{1}{r^{2+\varepsilon}}\right) \text { asymptotically } \\
& \left\langle\varepsilon^{3 i k} n_{i} g_{k j}\right\rangle \sim\left\langle\varepsilon^{3 i k} n_{i} \hat{g}_{k j}\right\rangle=\mathrm{O}\left(\frac{1}{r^{1+\xi}}\right) \text { asymptotically }
\end{aligned}
$$


and

$$
\left\langle\xi^{3 i k} n_{i} g_{k j} n_{l} g^{l j}{ }_{.0}\right\rangle-\left\langle\xi^{3 i k} n_{i} \hat{g}_{k j} n_{l} \hat{g}^{l j}{ }_{, 0}\right\rangle=\mathrm{O}\left(\frac{1}{r^{3+\varepsilon}}\right) \text { asymptotically. }
$$

Proof. $(a)+(c) \Rightarrow(b)$. For any region $\Xi$ and allowed perturbation $\delta$ we get from (3.23)

$$
\int_{\equiv}\left(G^{\alpha \beta}-8 \pi T^{\alpha \beta}\right)(-g)^{1 / 2} \mathcal{E}_{\xi} \delta g_{\alpha \beta}=0
$$

and since, apart from the restriction (3.8), $\delta g_{\alpha \beta}$ is free at every point, this implies $G^{\alpha \beta}-8 \pi T^{\alpha \beta}=0$.

$(b)+(c) \Rightarrow(a)$. As before, we obtain

$$
\int_{\equiv} \delta\left[\left(G^{\alpha \beta}-8 \pi T^{\alpha \beta}\right)(-g)^{1 / 2}\right] \mathfrak{E}_{\xi} g_{\alpha \beta}=0 .
$$

At any point we can choose $\delta g_{\alpha \beta}$ so that all ten $\delta\left[\left(G^{\alpha \beta}-8 \pi T^{\alpha \beta}\right)(-g)^{1 / 2}\right]$ are linearly independent, so that $\mathcal{E}_{\xi} g_{\alpha \beta}=0$ there.

$(a)+(b) \Rightarrow(c)$. Conditions $(a)$ and $(b)$ make the first two terms on the LHS of (3.23) vanish. Assume first that the variations are of compact support, then take a hypersurface $H^{\prime}$ away from this support and joining $H$ asymptotically, and let $\Xi$ be the region between the two hypersurfaces. Then the third term on the LHS of $(3.23)$ vanishes by restriction (1) in $(c)$, and the RHS reduces to $\delta P[\xi, H]$, which proves the theorem. Now, for variations with arbitrary support, theorem 3.2 shows that, subject to restrictions (2) and (3) in (c), these are equivalent on $H$ to variations of compact support, so that $P[\xi, H]$ remains an extremum.

Restriction (1) in (c) of theorem 3.3 becomes more transparent when not only the metric itself is axisymmetric, but also the energy-momentum of the matter, for if $\mathfrak{E}_{\xi} g_{\alpha \beta}=\mathfrak{L}_{\xi} T_{\alpha \beta}=0$ we can write

$$
\int_{\Xi} \xi^{\alpha} \delta\left(I_{\alpha ; \beta}^{\beta}\right) \mathrm{d}^{4} x=\oint_{\partial \Xi}\left(\delta I_{\alpha}^{\beta}-\frac{1}{2} I^{\mu \nu} \delta g_{\mu \nu} \delta_{\alpha}^{\beta}\right) \xi^{\alpha} \mathrm{d} \sigma_{\beta}
$$

and if $T_{\alpha \beta}$ is the energy-momentum of a perfect fluid

$$
T_{\beta}^{\alpha}=\mu n U^{\alpha} U_{\beta}+p \delta_{\beta}^{\alpha}
$$

then, further [1]

$$
\int_{\Xi} \xi^{\alpha} \delta\left(I_{\alpha ; \beta}^{\beta}\right) \mathrm{d}^{4} x=-\oint_{\partial \Xi}\left(\eta^{\nu} \delta V_{\nu} \delta_{\alpha}^{\beta}-\eta^{\beta} \delta V_{\alpha}-V_{\alpha} \delta \eta^{\beta}+n T(-g)^{1 / 2} \delta S \delta_{\alpha}^{\beta}\right) \xi^{\alpha} \mathrm{d} \sigma_{\beta}
$$

where $n$ is the number density of particles, $p$ the pressure, $\rho$ the mass-energy density, $\mu=(\rho+p) / n$ the relativisitic enthalpy, $S$ the specific entropy, $T$ the temperature, $\eta^{\alpha}=n(-g)^{1 / 2} U^{\alpha}$ the flux density, and $V_{\nu}=\mu U_{\nu}$ the momentum per particle of the fluid.

From the axisymmetry condition one may also write

$$
\begin{aligned}
\int_{\Xi} \frac{1}{16 \pi}\left(G^{\alpha \beta}-8 \pi T^{\alpha \beta}\right)(-g)^{1 / 2} \mathcal{E}_{\xi} \delta g_{\alpha \beta} \mathrm{d}^{4} x \\
=\frac{1}{16 \pi} \oint_{\partial \Xi}\left(G^{\alpha \beta}-8 \pi T^{\alpha \beta}\right)(-g)^{1 / 2} \delta g_{\alpha \beta} \xi^{\mu} \mathrm{d} \sigma_{\mu}
\end{aligned}
$$


and using (3.26) and $(3.27),(3.23)$ reduces to

$$
\begin{gathered}
-\oint_{\partial \Xi} \frac{1}{16 \pi}\left(G^{\alpha \beta}-8 \pi T^{\alpha \beta}\right)(-g)^{1 / 2} \delta g_{\alpha \beta} \xi^{\mu} \mathrm{d} \sigma_{\mu} \\
-\oint_{\partial \Xi}\left(\eta^{\nu} \delta V_{\nu} \delta_{\alpha}^{\beta}-\eta^{\beta} \delta V_{\alpha}-V_{\alpha} \delta \eta^{\beta}+n T(-g)^{1 / 2} \delta S \delta^{\beta}{ }_{\alpha}\right) \xi^{\alpha} \mathrm{d} \sigma_{\beta} \\
=\oint_{\partial \Xi} \delta\left[t_{N}{ }_{\nu}^{\mu} \cdot \xi^{\nu}+I^{\mu}{ }_{\nu} \xi^{\nu}\right] \mathrm{d} \sigma_{\mu}
\end{gathered}
$$

for any region $\Xi$. We then have the following theorem.

Theorem 3.4. Any axisymmetric spacetime containing a perfect fluid is a solution of Einstein's equations if and only if its angular momentum, measured on any asymptotically regular spacelike hypersurface $H$ containing $\xi$, is an extremum against all perturbations obeying

(a) $\xi^{\alpha} \delta V_{\alpha}=0$

(b) the number of particles is constant if $V_{\alpha} \xi^{\alpha} \neq 0$

(c) $\left\langle\varepsilon^{3 i k} n_{i} \delta g_{0 k}\right\rangle=\mathrm{O}\left(1 / r^{2+\varepsilon}\right)$ asymptotically

(d) $\left\langle\varepsilon^{3 i k} n_{i} g_{k j}\right\rangle \sim\left\langle\varepsilon^{3 i k} n_{i} \hat{g}_{k j}\right\rangle=\mathrm{O}\left(1 / r^{1+\varepsilon}\right)$

$$
\left\langle\varepsilon^{3 i k} n_{i} g_{k j} n_{l} g_{, 0}^{l j}\right\rangle \sim\left\langle\varepsilon^{3 i k} n_{i} \hat{g}_{k j} n_{i} \hat{g}_{, 0}^{l j}\right\rangle=\mathrm{O}\left(1 / r^{3+\varepsilon}\right) \text { asymptotically. }
$$

When the region under consideration extends out to infinity, the metric is assumed to have the asymptotic fall-off given by (3.2),

Proof. Assume first that the perturbations are of compact support, and arrange $\partial \Xi$ as in theorem 3.3 so that only $H$ contributes to the integrals in $(3.28)$, which we call $I_{1}$, $I_{2}$ and $I_{3}$ (in that order) for short. Now, since $\xi^{\mu}$ is contained in $H$, then $\delta^{\beta}{ }_{\alpha} \xi^{\alpha} \mathrm{d} \sigma_{\beta}=0$ and only two terms remain in the integrand of $I_{2}$, and these vanish for perturbations satisfying $(a)$ and $(b)$. Since $I_{3}$ is just the variation in the angular momentum, and since $\delta g_{\alpha \beta}$ is arbitrary at every point, it is immediate that the angular momentum is an extremum (i.e. $I_{3}$ vanishes) if and only if Einstein's equations are satisfied (i.e. $I_{1}$ vanishes).

Conditions $(c)$ and $(d)$ make the more general variations equivalent (as far as extremality is concerned) to those of compact support (cf theorem 3.2).

Comments. Restriction $(a)$ does not allow the perturbations to add momentum in the direction of $\xi$ (i.e. angular momentum) to the matter. Restriction (b) says that, if the angular momentum per particle is not zero, the perturbations should not alter the number of particles (thereby altering the total angular momentum to first order, as in the case of $(a)$ ). Restriction $(c)$, as seen already, is the equivalent of the above restrictions for the gravitational field. Restriction $(d)$ ensures that the angular momentum is well defined. The restrictions above are, then, what one intuitively expects must be restricted.

This theorem offers a different approach to calculating, say, axisymmetric gravitational collapse. Rather than solve the Einstein equations one can determine the solution by demanding its angular momentum be an extremum on every hypersurface. Such a method is harder to use than energy variational principles, because for energy the resulting variational solution is stationary, determined for all time by a variational calculation on one hypersurface. This difference can be traced back to the fact that, 
for energy, $\xi$ is transverse to $H$, while for angular momentum it lies in it. This provides motivation for studying extrema of the angular momentum flux, which we now do.

\section{Momentum flux across a timelike hypersurface}

To obtain the theorems in $\S 3$ for angular momentum, not only were stronger assumptions necessary on the metric coefficients, but also extra restrictions on the variations had to be introduced, as compared with the situation for energy. It seems natural then to ask, what is it about time as opposed to space that makes it easier to prove extremum theorems?

The most obvious difference in both approaches is that, whereas to measure energy one uses a timelike vector field $\xi_{E}$ which is transverse to the hypersurface $H$ of integration (the hypersurface containing all the fields), to measure angular momentum one uses a spacelike field $\xi_{\text {AM }}$ which is contained in $H$. This suggests that it would be easy to obtain an extremum theorem for the momentum flux $P[\xi, H]$ across a timelike hypersurface $H$, with $\xi=\partial / \partial \phi$, where the relation between surface and vector field is as in the case of energy. (Here $\phi$ is an azimuthal parameter with closed orbits.) We prove that this is indeed so.

Assume that the metric coefficients satisfy (3.2) in an ALCS $(T, x, y, z)$, and let $H=\{\phi=$ constant $\} \cap\left\{T \in\left[0, T_{0}\right]\right\}$ for a certain value $T_{0}>0$. We write, as before,

$P[\xi, H]=-\frac{1}{8 \pi} \int_{H}\left\{(-g)^{1 / 2} G^{\mu}{ }_{\nu} \xi^{\nu}-\frac{1}{2} \partial_{\alpha}\left(h^{\mu \alpha \nu \beta}{ }_{\beta} \xi_{\nu}(-g)^{-1 / 2}\right)\right\} \mathrm{d} \sigma_{\mu}$

where $\mathrm{d} \sigma_{\mu}$ is now the coordinate volume element of the timelike $H$. Under an asymptotic metric transformation of the form given by (3.4), this changes as (cf (3.6))

$P[\xi, H] \rightarrow P[\xi, H] \underset{\substack{r=R \\ T \in\left[0, T_{0}\right]}}{ }+\int_{\substack{r>R \\\left[0, T_{0}\right]}} \frac{1}{16 \pi} h_{, \beta}^{\mu \alpha \nu \beta}(-g)^{-1 / 2} \xi_{\nu, \alpha} \mathrm{d} \sigma_{\mu}$.

Writing $g_{\mu \nu}=\eta_{\mu \nu}+h_{\mu \nu},(3.4)$ implies (we do not write indices)

$$
\partial h \rightarrow \partial h+(t \partial h+h \partial t)
$$

and so the integrand in (4.2) transforms into $\eta \partial h+\eta t \partial h+\eta h \partial t+$ lesser terms, and a simple order-of-magnitude analysis shows that the integral vanishes as $R \rightarrow \infty$, so that $P[\xi, H]$ is unaltered under an asymptotic change of metric, for a finite time interval.

Theorem 4.1. Let $H$ be a timelike hypersurface transverse to $\partial / \partial \phi$, that is asymptotically regular in the spatial directions. Let $\xi^{\mu}=x \delta_{2}^{\mu}-y \delta_{1}^{\mu}$ asymptotically in an ALCS. Then, any solution of Einstein's equations is an extremum of the momentum flux across $H$ measured by $\xi$, against all variations of finite time duration.

This variational principle has more teeth. Here we can study axisymmetric collapse in a radically different way. On a surface $\phi=$ constant, we guess the time behaviour of the metric and fluid. If and only if the resultant flux integral is an extremum against perturbations of finite duration will the guess be a solution of Einstein's equations.

The variations of the field variables are required to be of finite time duration because the spacetime is asymptotically flat in the spatial directions, but not in the time direction. However, a real perturbation satisfying Einstein's equations will not go away after a finite amount of time. It would be interesting to see what conditions this requirement would lead to. 


\section{Conclusions}

We have shown how one can have a considerably enhanced flexibility in the use of pseudotensors for conserved quantities, since spherical coordinate systems are the natural frames to use for isolated systems. We have developed and proved extremum theorems for the angular momentum of solutions of Einstein's equations. These theorems may be very useful in showing that a given metric solves the field equations, without having to solve the equations themselves. They can also help with the very construction of spacetime metrics. The feasibility of implementing these techniques to interesting self-gravitating systems is under study.

\section{Acknowledgment}

E N-A would like to thank the National University of Mexico for financial assistance.

\section{References}

[1] Schutz B F and Sorkin R 1977 Ann. Phys., NY 1071

[2] Trautman A 1962 Gravitation: an Introduction to Current Research ed L Witten (New York: Wiley) pp 169-98

[3] Nahmad-Achar E and Schutz B F 1987 Gen. Rel. Grav. in press

[4] Penrose R 1982 Proc. R. Soc. A 38153

[5] Einstein A 1916 Ann. Phys., Lpz 49769

[6] Landau L D and Lifshitz E M 1951 The Classical Theory of Fields (Reading, MA: Addison Wesley)

[7] Tod K P 1983 Proc. R. Soc. A 388457

[8] Choquet-Bruhat Y and York J W Jr 1980 General Relativity and Gravitation ed A Held (New York: Plenum) p 99 